\title{
Re: Baek et al Postoperative Longitudinal Outcomes in Patients with Residual Disc Fragments after Percutaneous Endoscopic Lumbar Discectomy
}

\section{TO THE EDITOR:}

We read with interest the article "Postoperative longitudinal outcomes in patients with residual disc fragments after percutaneous endoscopic lumbar discectomy" by Baek et al (1). The authors first compared the longitudinal clinical outcomes of percutaneous endoscopic lumbar discectomy (PELD) patients to the complete group and the residual group. However, it is really disputable that they would divide patients into groups using the magnetic resonance imaging (MRI) within 24 hours postoperatively. Different from the traditional open or microendoscopic discectomy, PELD is performed under continuous saline irrigation. The evaluation of neural decompression is likely to be affected by residual fluid and the adjacent edematous tissue, especially within 24 hours postoperatively. In the presented case, preoperative sagittal T2-weighted MRI showed a herniated lumbar disc that migrated inferiorly from L5-S1. The "residual disc fragments" remained at S1 at the MRI immediately following the procedure, which was completely resorbed at the 6-month followup MRI (Fig. 1).

Is it possible that the so-called "residual disc fragments" are residual fluid or edematous tissue? If so, it should be declared in the limitation section.

In order to avoid interference from residual fluid, the sagittal T1-weighted MRI seems to be more appropriate to detect the residual compression from the prolapsed nucleus pulposus at the early stage postoperatively.

Jun-Song Yang, MD

Department of Spine Surgery

Honghui Hospital

Xi'an Jiaotong University,

No. 76 Nanguo Road, Xi'an

Shaanxi, 710054, China

Kai-Xuan Liu, MD

Atlantic Spine Center

475 Prospect Avenue, Suite 110

West Orange, NJ 07052, USA

Hong Fan, MD

Department of Spine Surgery

Honghui Hospital

Xi'an Jiaotong University,
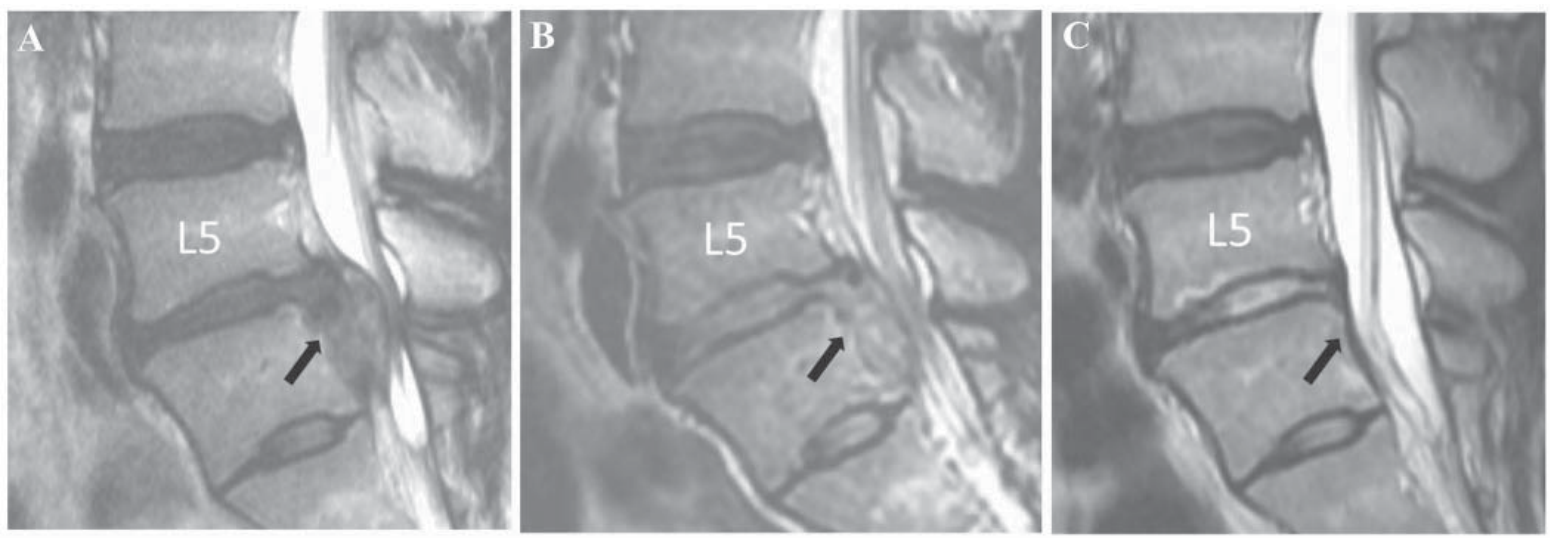

Fig. 1. Preoperative sagittal T2-weighted MRI showed a herniated lumbar disc that migrated inferiorly from L5-S1 (A, arrow). And the 'residual disc fragments' remained at $S 1$ (B, arrow) at the MRI immediately after operation, which was completely resorbed at the 6-month follow-up MRI ( $C$, arrow). 
No. 76 Nanguo Road, Xi'an Shaanxi, 710054, China

Tuan-Jiang Liu, MD

Department of Spine Surgery

Honghui Hospital

Xi'an Jiaotong University,

No. 76 Nanguo Road, Xi'an

Shaanxi, 710054, China

Peng Liu, MD

Department of Spine Surgery

Honghui Hospital

\section{References}

1. Baek J, Yang $\mathrm{SH}$, Kim $\mathrm{CH}$, Chung $\mathrm{CK}$, Choi Y, Heo JH, Park SB, Hwang SH, Jung JM, Kim KT. Postoperative longitudinal outcomes in patients with residual
Xi'an Jiaotong University, No. 76 Nanguo Road, Xi'an Shaanxi, 710054, China

Ding-Jun Hao, MD

Department of Spine Surgery

Honghui Hospital

Xi'an Jiaotong University, No. 76 Nanguo Road, Xi'an Shaanxi, 710054, China

E-mail: dingjun.hao@qq.com disc fragments after percutaneous endoscopic lumbar discectomy. Pain Physician 2018; 21:E457-E466. 


\section{In Response to Letter Re: Postoperative Longitudinal Outcomes in Patients with Residual Disc Fragments}

I appreciate the important comment supplied by Dr. Hao et al for our manuscript (1). We took an MRI within 24 hours because most patients were discharged one day after surgery. I agree with your opinion that the so-called "residual disc fragments" was not a sole residual disc fragment but mixed tissue with residual fluid and edematous tissue, but the differentiation of those was not simple even with T1-weighted magnetic resonance imaging (MRI) (Fig. 1a and b). Nonetheless, some patients were symptomatic due to persistent symptoms of the mixed tissue, and removal of the remaining disc fragment relieved the symptoms. This finding suggested that the mixed tissue contained residual disc fragments. The main take-away message is that "watchful waiting" is a sufficient strategy for asymptomatic patients, even with persistent compression by a mixed tissue with a residual disc, retained fluid and/or edematous tissue.

Chi Heon Kim, MD, PhD

Department of Neurosurgery,

Seoul National

University College of Medicine

101 Daehak-Ro, Jongno-gu

Seoul, 110-744, South Korea

E-mail: chiheon1@snu.ac.kr
Fig 1. T1-weightd pre and postoperative images

Preoperative sagittal T1-weighted magnetic resonance imaging (MRI) presenting a herniated intervertebral disc (arrow) that has inferiorly migrated from L5-S1 (a). Immediate postoperative MRI demonstrated that decompression was achieved, but the mixed tissue (arrow) still compressed the dura (b).
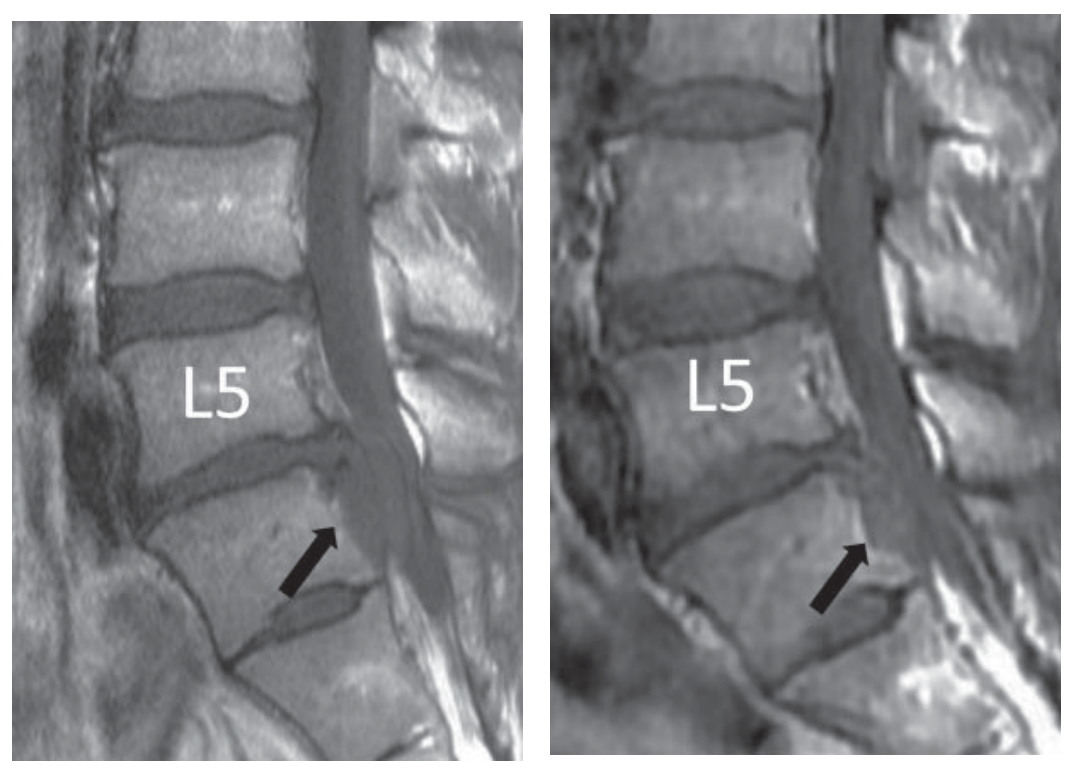

\section{REFERENCES}

1. Baek J, Yang $\mathrm{SH}$, Kim CH, Chung $\mathrm{CK}$, Choi Y, Heo JH, Park SB, Hwang SH, Jung JM, Kim KT. Postoperative longitudinal outcomes in patients with residual disc fragments after percutaneous endoscopic lumbar discectomy. Pain Physician 2018:21: E457-E466. 